\title{
Quantitative Trait Loci Influencing Cholesterol and Phospholipid Phenotypes Map to Chromosomes That Contain Genes Regulating Blood Pressure in the Spontaneously Hypertensive Rat
}

\author{
Anita Bottger, ${ }^{\star}$ Hein A. van Lith, ${ }^{\star}$ Vladimír Kren, ${ }^{\ddagger}$ Drahomíra Krenová, ${ }^{\ddagger}$ Vlasta Bílá, ${ }^{\ddagger}$ Jaroslav Vorlícek, ${ }^{\S}$ Václav Zídek, ${ }^{\S}$ \\ Alena Musilová, $\$$ Miroslava Zdobinská, $\$$ Jia-Ming Wang, Bert F.M. van Zutphen, ${ }^{*}$ Theodore W. Kurtz,\| and Michal Pravenec $\$$ \\ $*$ Department of Laboratory Animal Science, Veterinary Faculty, Utrecht University, 3508 TD Utrecht, The Netherlands; ${ }^{\ddagger}$ Department of \\ Biology, 1st Medical Faculty, Charles University, 12800 Prague, Czech Republic; ${ }^{\S}$ Institute of Physiology, Czech Academy of Sciences,

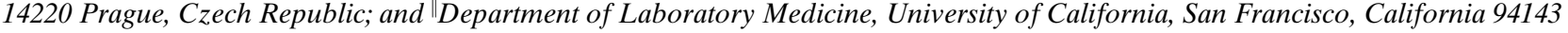

\begin{abstract}
The frequent coincidence of hypertension and dyslipidemia suggests that related genetic factors might underlie these common risk factors for cardiovascular disease. To investigate whether quantitative trait loci (QTLs) regulating lipid levels map to chromosomes known to contain genes regulating blood pressure, we used a genome scanning approach to map QTLs influencing cholesterol and phospholipid phenotypes in a large set of recombinant inbred strains and in congenic strains derived from the spontaneously hypertensive rat and normotensive Brown-Norway (BN.Lx) rat fed normal and high cholesterol diets. QTLs regulating lipid phenotypes were mapped by scanning the genome with $\mathbf{5 3 4}$ genetic markers. A significant relationship $(P<0.00006)$ was found between basal HDL2 cholesterol levels and the D19Mit2 marker on chromosome 19. Analysis of congenic strains of spontaneously hypertensive rat indicated that QTLs regulating postdietary lipid phenotypes exist also on chromosomes 8 and 20. Previous studies in the recombinant inbred and congenic strains have demonstrated the presence of blood pressure regulatory genes in corresponding segments of chromosomes 8,19 , and 20 . These findings provide support for the hypothesis that blood pressure and certain lipid subfractions can be modulated by linked genes or perhaps even the same genes. (J. Clin. Invest. 1996. 98:856862.) Key words: rat • genetics • dyslipidemia • hypertension - quantitative trait loci
\end{abstract}

\section{Introduction}

Epidemiologic studies have demonstrated that high blood pressure is often associated with dyslipidemia and it has been proposed that essential hypertension may represent one element of a complex metabolic syndrome. Both hypertension and dyslipidemia constitute important risk factors for coronary

Address correspondence to Dr. Michal Pravenec, Institute of Physiology, Czech Academy of Sciences, Vídeňská 1083, 14220 Prague 4, Czech Republic. Phone: 422-475-2297; FAX: 422-471-9517; E-mail: pravenec@biomed.cas.cz

Received for publication 21 February 1996 and accepted in revised form 10 June 1996.

J. Clin. Invest.

(C) The American Society for Clinical Investigation, Inc. 0021-9738/96/08/0856/07 \$2.00

Volume 98, Number 3, August 1996, 856-862 heart disease and together contribute to clinical disorders such as Syndrome X (1-3) and familial dyslipidemic hypertension $(\mathrm{FDH})^{1}(4,5)$. Dyslipidemia associated with Syndrome $\mathrm{X}$ is manifested by high plasma triglycerides, high concentrations of VLDL and LDL fractions, and low HDL-cholesterol concentrations. Similar findings have been reported in individuals with FDH. The coincidence of hypertension and dyslipidemia could stem from environmental factors such as excess fat intake or obesity that promote both increased blood pressure and disordered lipid levels. In addition, the frequent association of hypertension and dyslipidemia might be influenced by genes that regulate blood pressure and lipid metabolism. For example, to the extent that insulin resistance mediates increased blood pressure and disordered lipid metabolism (1), genetic variants that impair insulin sensitivity could promote both hypertension and hyperlipidemia. Based on extensive genetic studies in patients with FDH, Williams and colleagues (5) have raised the interesting possibility that a gene linked to the regulation of LDL subfractions could also be a candidate gene for hypertension. Alternatively, the existence of linked genes that independently influence blood pressure and insulin resistance or lipid metabolism could also explain the clustering of metabolic risk factors in hypertension.

In human essential hypertension, the detailed genetic analysis of multifactorial metabolic syndromes is very complicated. Therefore, it is hoped that studies of animal models might shed light on genetic mechanisms underlying dyslipidemia and high blood pressure. Recent studies have suggested that increased blood pressure may be associated with disorders of lipid and or insulin metabolism in several animal models of hypertension including the spontaneously hypertensive rat (SHR) (6), the Dahl salt-sensitive rat (7), the Milan rat (8), the Lyon rat (9), and the fructose fed rat (10). Thus, rodent models might be useful for investigating whether genes regulating blood pressure are linked to genes influencing lipid metabolism. The availability of a large set of extensively genotyped recombinant inbred (RI) strains derived from the SHR and the normotensive BN.Lx rat (HXB and BXH RI strains) provides a unique opportunity for mapping genes influencing blood pressure and serum lipid levels in a commonly used animal model of hypertension. In previous genetic studies in these RI strains and in congenic strains derived from SHR and normotensive Brown-Norway (BN.Lx) rats, we identified several chromo-

1. Abbreviations used in this paper: BN.Lx, normotensive BrownNorway rats; FDH, familial dyslipidemic hypertension; QTLs, quantitative trait loci; RI, recombinant inbred; SHR, spontaneously hypertensive rat. 
some regions that contain quantitative trait loci (QTLs) regulating blood pressure $(11,12)$. In the current studies in the HXB and BXH RI strains, we investigated whether genes influencing lipid metabolism map to any of these chromosome regions known to contain genes regulating blood pressure. Specifically, we used a genome scanning approach to map QTLs regulating cholesterol and phospholipid phenotypes in the HXB and BXH RI strains and compared their map locations with those of previously mapped blood pressure QTLs. We have found evidence that QTLs influencing HDL2 cholesterol and phospholipid levels map to three distinct regions previously reported to contain QTLs regulating blood pressure. The current findings provide support for the hypothesis that blood pressure and lipid levels can be modulated by linked genes or perhaps even the same genes and are consistent with the idea that genetic factors may contribute to the frequent association of dyslipidemia and hypertension.

\section{Methods}

\section{Animals}

Derivation of RI strains. The RI strains were derived from spontaneously hypertensive rats (SHR/Ola) and normotensive Brown-Norway rats $(\mathrm{BN} . \mathrm{Lx} / \mathrm{Cub})(11)$. The BN.Lx progenitor is a congenic strain that carries a segment of chromosome 8 from the polydactylous PD/ Cub strain (13). $36 \mathrm{RI}$ strains were originally obtained from crosses of female SHR and male BN.Lx rats (HXB strains, $n=26$ ) or female BN.Lx rats and male SHR (BXH strains, $n=10$ ). Of the 36 original strains, 32 surviving strains are still available (22 HXB strains and 10 BXH strains) and are currently beyond F35. DNA samples were available from all 36 of the original strains. In the current study, $30 \mathrm{RI}$ strains have been used (HXB strains, $n=21$ and BXH strains, $n=9$ ). In each RI strain, three to six males were tested for cholesterol and phospholipid phenotypes.

Derivation and genetic analysis of SHR.Lx and SHR.1N congenic strains. The SHR.1N congenic strain was derived by transferring a segment of chromosome 20 from the BN.Lx strain onto the genetic background of the SHR strain (11). Animals of N14F22 generation were used in the current study. The SHR.Lx congenic strain was derived by transferring the chromosome 8 segment with the $L x$ allele (coding for the polydactyly-luxate syndrome originally described in the polydactylous PD rat) from the BXH11 RI strain by 11 backcrosses with the SHR/Ola strain (14). Since the progenitor SHR $(+/+)$ and heterozygous SHR $(L x /+)$ animals are normodactylous, the heterozygotes in each backcross generation were genotyped by mating with polydactylous homozygotes $(L x / L x)$. The SHR.Lx congenic strain carries a chromosome 8 segment of PD origin that is flanked on both sides by BN chromosome segments. The PD strain is normotensive similarly as the BN.Lx strain, but it is hypertriglyceridemic (15). The animals of N11F3 generation were used in the current study. The congenic status of SHR.Lx and SHR.1N strains has been confirmed by testing with the following microsatellite loci: D1Mit9, D1Mit14, Igf2, and Pthr1 (chr.1); D2Mit4, D2Mit16, D2N91, and Atp1a1 (chr.2); D3Mit5, D3Mit10, and D3Mit11 (chr.3); Eno2, Il6, and Spr (chr.4); D5Mgh8, D5Mit1, and Slc2a1 (chr.5); D6Cep8, D6Mit8, D6Mit9, and Ighe (chr.6); D7Mgh11, D7Mit6, and D7Mit8 (chr.7); D8Mgh4, D8Mgh5, D8Mgh6, D8Mgh7, D8Mgh9, D8Mit1, D8Mit4, D8Mit5, D8Mit6, D8Mit12, D8N228, Acaa, Apoc3, Es6, Lx, Ncam, Rbp2, Thy1, and Tpm1 (chr.8); D9Mit1, D9Mit4, and Inha (chr.9); D10Mit6, Abpa, Dcp1, and Myh3 (chr.10); D11Mgh4, D11Mgh6, and Sst (chr.11); D12Mgh4, D12Mit8, and Mdh2 (chr.12); D13Mgh3, D13Mit2, and D13Mit4 (chr.13); D14Mgh1, D14Mit1, D14Mit8, and Alb (chr.14); D15Mgh3, D15Mgh5, and D15Mit3 (chr.15); D16Mit2, D16Mit3, and Mbpa (chr.16); D17Mit2, D17Mit7, and Chrm3 (chr.17); D18Mit1, D18Mit10, and Grl (chr.18); D19Mit2, D19Mit5, and D19Mit7 (chr.19); D20Arb249, D20Arb548, D20Mgh1, D20Mgh2, D20Utr1,
D20Utr2, Rt1a, and Tnfa (chr.20); DXMgh1, DXMit5, and Arl (chr.X). PCR primers were obtained from Research Genetics (Huntsville, $\mathrm{AL})$ or synthesized in the UCSF Biomolecular Resource Center according to published sequences (16-19).

\section{Experimental diet}

After weaning until the age of $7 \mathrm{wk}$, the animals were fed a commercial pelleted diet (RMH-B, Hope Farms BV, Woerden, The Netherlands). After this preexperimental period, the rats were fed for $4 \mathrm{wk}$ the commercial diet supplemented with $5.0 \%$ (wt/wt) olive oil (Reddy, Van de Moortele NV., Oudenbosch, The Netherlands) and $2.0 \%$ (wt/wt) cholesterol (USP, Solvay/Duphar BV, Weesp, The Netherlands). Food and tap water were available ad libitum. The experimental diet was provided in pelleted form and was stored in the freezer until use. The chemical composition of the commercial diet has been described previously (20).

\section{Preparation of samples and chemical analysis}

Blood samples were taken at random order between 08.00 and 10.00 $\mathrm{h}$ after a 16-h fasting period. Blood was collected under light diethyl ether anesthesia from the tail vein. Sera of the animals were cooled at $4^{\circ} \mathrm{C}$ and kept at that temperature until ultracentrifugation. Serum total cholesterol and total phospholipid concentrations were determined using enzymatic test kits (Boehringer-Mannheim $\mathrm{GmbH}$, Mannheim, Germany). For each RI strain, the aliquots of sera from all animals were pooled and the lipoprotein fractions were isolated by density gradient ultracentrifugation (centrifuge effect $(\omega)^{2} t=$ $1.39 .10^{12} \mathrm{rad}^{2} / \mathrm{s}$ ) as described (21), except for staining. Isolation of the lipoprotein fractions was done within $5 \mathrm{~d}$ after blood sampling. Lipoprotein density classes were based on the pattern observed in humans: VLDL $(d<1.006)$, IDL $(1.006<d<1.019)$, LDL/HDL1 $(1.019<d$ $<1.063)$, HDL2 $(1.063<d<1.125)$. The lipoprotein cholesterol and phospholipid concentrations in each fraction were determined using enzymatic test kits (Boehringer-Mannheim $\mathrm{GmbH}$ ). Lipoprotein cholesterol and phospholipid concentrations were corrected for recovery.

\section{Derivation of genetic linkage map of RI strains}

We analyzed the RI strain distribution patterns for a total of 534 biochemical, morphologic, immunogenetic, and molecular genetic markers (microsatellite polymorphisms typeable by PCR and microsatellite/minisatellite polymorphisms typeable by Southern blot/DNA fingerprint analysis). Typing methods and strain distribution patterns for some of these markers have been described previously (22). Molecular genetic markers consisting of amplified fragment length polymorphisms (AFLPs) were also used (23).

RFLP of the PCR products digested with the BglII enzyme distinguished the alleles of the lipoprotein lipase $(L p l)$ gene between the BN.Lx and SHR strains. The primers were designed according to the published sequence (24): the upstream primer was 5'-TGC CCA ATC GTT AGC ATT TC, the downstream primer was 5'-GAT CCC ATT TCT TCA CAC AG. The size of the uncut PCR product was 957 bp. After digestion with the BglII restriction endonuclease, two bands were detected: in SHR, the band sizes were $\sim 316$ and $641 \mathrm{bp}$, whereas in BN.Lx the band sizes were $\sim 320$ and 637 bp. The Lcat gene, coding for lecithin cholesterol acyl transferase, has been mapped by finding an RFLP of the PCR products between SHR and BN.Lx strains. The primers were designed according to the published cDNA sequence (25) to amplify the last intron of the Lcat gene as based on homology with the human complete DNA sequence (26): the upstream primer was 5'-TGA TGG CTT CAT CTC TCT CG, the downstream primer was 5 '-AGG GGG AAG TTG TGG TTA TG. The size of the uncut PCR product was $\sim 1,350 \mathrm{bp}$. The RFLP between BN.Lx and SHR strain was detected by cutting the PCR product with the NlaIII enzyme: the BN.Lx DNA contained the NlaIII restriction site, the SHR DNA did not. The Map Manager program of Manly (version 2.6.5.) was used to estimate the map location of the $L p l$ and $L c a t$ genes (27). 


\section{QTL analysis}

For testing of possible associations of 534 genetic markers with lipoprotein cholesterol and phospholipid phenotypes, we used a special computer program that performed automatically two-tailed $t$ tests over all markers and all phenotypes. Since the genome scanning approach involves the testing of multiple markers and phenotypes, it is necessary to use stringent statistical criteria in the linkage analysis. Belknap and Neumann have suggested statistical guidelines for genome scanning analysis of RI strains that are based on a Bonferroni correction for multiple comparisons. For the Bonferroni adjustment, the number of independent tests is considered to be a function of the genomic distance swept by a given set of RI strains. In a set of $30 \mathrm{RI}$ strains, a Bonferroni adjusted $P$ value $<0.0006$ corresponds approximately to a type I error of $<0.05(28,29)$. We have further corrected this criterion of statistical significance for simultaneous testing of multiple phenotypes. In this study, we tested 10 phenotypes: total cholesterol and phospholipids, VLDL cholesterol and phospholipids, LDL/HDL1 cholesterol and phospholipids, IDL cholesterol and phospholipids, and HDL2 cholesterol and phospholipids. Accordingly, in the RI strain analysis of lipid phenotypes, we have defined the statistical significance as $P<0.00006(0.0006 / 10=0.00006)$. A Bonferroni correction has also been applied for the simultaneous comparisons between the progenitor strain and the congenic strains with respect to 10 lipid phenotypes: an adjusted $P$ value of 0.005 $(0.05 / 10=0.005)$ was used as the cutoff for statistical significance.

\section{Results}

Cholesterol and phospholipid phenotypes of progenitor strains. When fed the control diet, both the SHR and BN.Lx progenitor strains had similar profiles of serum cholesterol and phospholipid phenotypes (data not shown). However, as can be seen in Table I, significant differences were observed between BN.Lx and SHR rats after feeding the high fat and high cholesterol diet for $4 \mathrm{wk}$ : the BN.Lx rats had significantly increased serum concentrations of total cholesterol and phospholipids which were mainly due to the increase in VLDL cholesterol and phospholipid fractions. On the other hand, HDL2 values decreased in both progenitors.

Cholesterol and phospholipid phenotypes in RI strains. Phenotyping of RI strains showed that the distributions of cholesterol concentrations in total serum and in lipoprotein fractions were continuous and in most cases significantly different from the midparental values (Fig. 1, $A$ and $B$ ). Similar distribu-
A

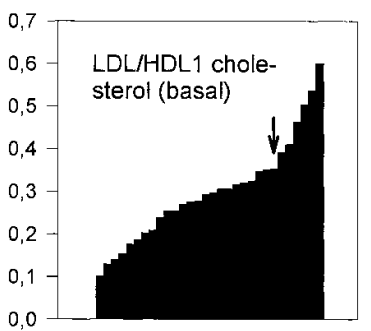

B

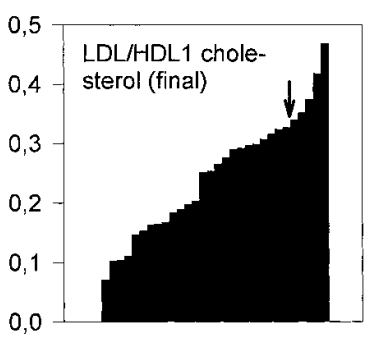

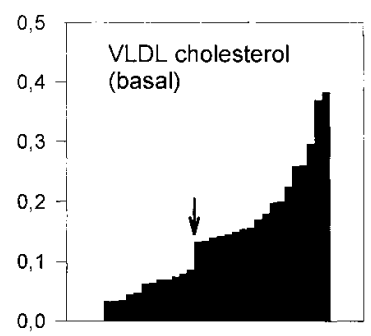
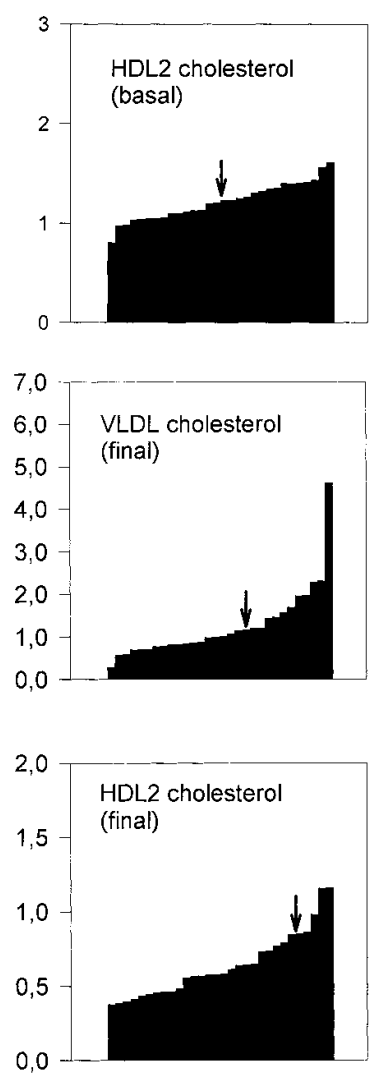

Figure 1. Distributions of cholesterol concentrations (in $\mathrm{mmol} / \mathrm{liter}$ ) in total serum and in selected lipoprotein fractions among RI strains. Basal values were obtained in rats fed a control diet $(A)$; final values were obtained in rats fed a diet with high fat and high cholesterol content $(B)$. Arrows indicate the midparental values for each phenotype. 
Table I. Cholesterol and Phospholipid Levels in Total Serum and in Lipoprotein Fractions in BN.Lx $(n=4)$ and SHR $(n=5)$ Progenitor Strains Fed a Diet with 5\% Olive Oil and $2 \%$ Cholesterol

\begin{tabular}{lccccc}
\hline & \multicolumn{2}{c}{ Cholesterol (mmol/liter) } & & \multicolumn{2}{c}{ Phospholipids (mmol/liter) } \\
\cline { 2 - 3 } \cline { 6 - 6 } & BN.Lx & SHR & & BN.Lx & SHR \\
\hline Total & $3.13 \pm 0.06$ & $2.01 \pm 0.07^{*}$ & & $1.51 \pm 0.03$ & $1.31 \pm 0.03^{\ddagger}$ \\
VLDL & $1.47 \pm 0.10$ & $0.81 \pm 0.11^{\ddagger}$ & & $0.40 \pm 0.02$ & $0.20 \pm 0.03^{\ddagger}$ \\
IDL & $0.33 \pm 0.03$ & $0.32 \pm 0.03$ & & $0.20 \pm 0.03$ & $0.08 \pm 0.01$ \\
LDL/HDL1 & $0.37 \pm 0.05$ & $0.29 \pm 0.03$ & & $0.11 \pm 0.02$ & $0.08 \pm 0.01$ \\
HDL2 & $0.85 \pm 0.01$ & $0.73 \pm 0.03$ & & $0.20 \pm 0.03$ & $0.45 \pm 0.01^{*}$
\end{tabular}

${ }^{\ddagger} P<0.005, * P<0.0001$ (two-tailed Student $t$ test), all values are expressed as means \pm SEM.

tions were detected in phospholipid concentrations (data not shown).

Detection of QTLs contributing to cholesterol and phospholipid phenotypes in RI strains. Table II and Fig. 2 show the results of scanning of the genome of RI strains with 534 gene markers. These markers are randomly distributed throughout the genome of RI strains and cover $\sim 1,200 \mathrm{cM}(22,23)$. A significant association $(P<0.00006)$ was found between the basal HDL2 cholesterol level and the D19Mit2 marker on chromosome 19. The D19Mit2 marker was the only marker that achieved the stringent level of statistical significance required for linkage. Several suggestive associations were also detected between selected lipid phenotypes and markers on chromosomes 4 and 20 although these did not achieve the level of statistical significance required to indicate linkage; the basal level of HDL2 phospholipids correlated with the Il6 marker on chromosome $4(P<0.0005)$ and the postfeeding HDL2 levels correlated with D20Utrl on chromosome $20(P<0.00035)$. These markers on chromosomes 4,19 , and 20 map near corresponding chromosome regions previously implicated in genetic studies of blood pressure in RI strains $(11,12)$.

Mapping of Lcat and Lpl genes in RI strains. Based on the homology with the mouse, the D19Mit2 marker which showed the strongest association with basal HDL2 cholesterol was ex-

Table II. Associations of Gene Markers with Basal and Final Cholesterol and Phospholipid Levels in Total Serum and in Lipoprotein Fractions in RI Strains (Only the Most Pronounced Associations Are Shown)

\begin{tabular}{llllll}
\hline \multicolumn{1}{c}{ Variable } & Marker & BN.Lx allele & SHR allele & $P$ value & chr. \\
\hline Basal data & & & & & \\
HDL2 cholesterol & D19Mit2 & $1.35 \pm 0.10$ & $1.11 \pm 0.17$ & $0.00006^{*}$ & 19 \\
HDL2 phospholipids & II6 & $0.69 \pm 0.08$ & $0.54 \pm 0.08$ & 0.0005 & 4 \\
Final data & & & & & \\
HDL2 phospholipids & D20Utr1 & $0.38 \pm 0.12$ & $0.21 \pm 0.11$ & 0.00035 & 20 \\
Total phospholipids & D20Utr1 & $1.28 \pm 0.22$ & $0.96 \pm 0.18$ & 0.0002 & 20
\end{tabular}

*Significant association of the marker with a given phenotype; basal data were obtained on a control diet, final data on a diet supplemented with $5 \%$ olive oil and $2 \%$ cholesterol.

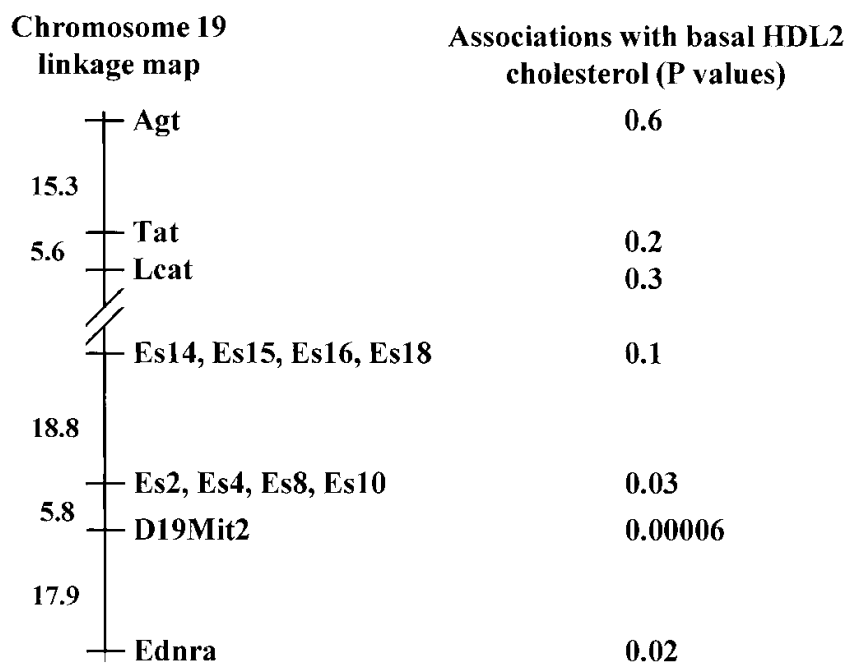

Figure 2. Linkage map of chromosome 19 with the D19Mit2 marker and the Lcat and esterase positional candidate genes. The numbers on the left of the map show the most likely distances between loci in centimorgans. The significance values ( $P$ values) from the $t$ statistics that indicate the strength of associations of markers with the basal HDL2 cholesterol are listed opposite each marker.

pected to be located in the vicinity of two positional candidate genes, Lpl (lipoprotein lipase) and Lcat (lecithin cholesterol acyl transferase) (30). However, in the rat, we were able to map the $L p l$ gene to chromosome 16 which excludes lipoprotein lipase as a candidate gene for the observed variation in basal HDL2 cholesterol; the estimated map location of chromosome 16 markers is: ...D16Mit3 - 4.4 (1.3 - 13.2) - D16Mit1 3.3 (0.8 - 10.7) - D16Mit5 - 0.0 (0.0 - 2.9) - Mbpa - 4.5 (1.1 16.0 ) - Lpl... (distances between loci and $95 \%$ confidence limits are in centimorgans, see reference 27). On the other hand, the Lcat gene has been mapped to chromosome 19; the most likely gene order is: ...Agt - 2.9 (0.3 - 15.1) - D19Cebr204s28 - 7.7 (1.7 - 36.0) - D19Mit7 - 4.7 (1.3 - 14.5) - Tat - 5.6 (1.3 - 23.0) - Lcat... (distances between loci and 95\% confidence limits are in centimorgans, see reference 27) (Fig. 2).

Detection of QTLs contributing to lipoprotein phenotypes in SHR. Lx and SHR.1N congenic strains. Molecular genetic analysis of SHR.Lx and SHR.1N strains with multiple microsatellite markers (at least three markers per each autosome and the $\mathrm{X}$ chromosome) confirmed their congenic status. Based on the map of Jacob et al. (17), the minimal length of the differential chromosome segment was estimated to be $\sim 35.2 \mathrm{cM}$ in SHR.Lx (the breakpoints separating the differential segment of chromosome 8 were between D8Mit6 and D8Mgh 9 markers and Cyp45c and Tpm1 markers, respectively). In SHR.1N strain, the length of the differential chromosome segment was estimated to be $\sim 31 \mathrm{cM}$, spanning the region of chromosome 20 between D20Cebrp215s7 and D20Utr1 markers, including, in addition, the swept distance covered by the D20Arb548 marker that could not be linked in the RI strains but is of $\mathrm{BN} /$ BN genotype in the SHR.1N strain (our unpublished results). Table III shows that the SHR.1N strain had significantly decreased LDL/HDL1 serum cholesterol concentrations and significantly increased HDL2 phospholipid concentrations when compared with the SHR progenitor strain. The SHR.Lx congenic strain also showed a decrease in final levels of LDL/HDL1 
Table III. Comparisons of SHR.Lx and SHR.1N Congenic Strains with the SHR Progenitor

\begin{tabular}{lcll}
\hline \multicolumn{1}{c}{ Phenotypes } & \multicolumn{1}{c}{ SHR } & \multicolumn{1}{c}{ SHR.Lx } & \multicolumn{1}{c}{ SHR.1N } \\
\hline LDL/HDL1 cholesterol & $0.29 \pm 0.03$ & $0.16 \pm 0.03$ & $0.20 \pm 0.01^{*}$ \\
IDL cholesterol & $0.23 \pm 0.03$ & $0.10 \pm 0.05^{*}$ & $0.13 \pm 0.02$ \\
HDL2 cholesterol & $0.73 \pm 0.03$ & $0.93 \pm 0.03^{\ddagger}$ & $0.92 \pm 0.05$ \\
HDL2 phospholipids & $0.31 \pm 0.01$ & $0.44 \pm 0.01^{\ddagger}$ & $0.44 \pm 0.02^{\ddagger}$
\end{tabular}

All results shown here are final values after feeding the diet with high content of fat and cholesterol. *Statistical significance $P<0.005$; *statistical significance $P<0.0005$. Other phenotypes tested in this study suggested no difference between the congenic strains and SHR. All values are mean \pm SEM.

cholesterol and an increase in postfeeding HDL2 cholesterol and phospholipids when compared with the SHR strain. Since the SHR.Lx and SHR.1N congenic strains were shown in previous studies to have significantly decreased blood pressure when compared with SHR $(11,31)$, the current findings indicate that both blood pressure regulatory gene(s) and QTL(s) modulating cholesterol and phospholipid variables map within the differential segments of chromosomes 8 and 20 in these congenic strains. Lower blood pressure in both congenic strains is associated with lower levels of the LDL/HDL1 fraction and higher levels of the HDL2 fraction.

\section{Discussion}

To detect QTLs responsible for selected cholesterol and phospholipid phenotypes, we scanned the genome of RI strains with a total of 534 gene markers. As can be seen in Table II and Fig. 2, basal HDL2 cholesterol levels were found to be strongly associated with the D19Mit2 marker on chromosome $19(P<0.00006)$. The RI strains that inherited this region of chromosome 19 from the SHR had lower levels of HDL2 than the strains that inherited the corresponding chromosome region from the normotensive Brown-Norway rat. Comparative gene mapping in humans, mice, and rats has revealed evidence for considerable conservation of gene order during mammalian evolution: the segment of the rat chromosome 19 containing D19Mit2 appears to be homologous to a segment of mouse chromosome 8 (32) and human chromosome 16 (33). This region of mouse chromosome 8 contains genes encoding lecithin cholesterol acyl transferase (Lcat) and lipoprotein lipase $(\mathrm{Lpl})$ (34-36). In the rat, we have mapped the $L p l$ gene to chromosome 16 which excludes it as a candidate gene for the observed variation in basal levels of HDL2 cholesterol. Also the Lcat gene maps a considerable distance away from D19Mit2, suggesting that it is not the QTL on chromosome 19 that influences HDL2 levels. On the other hand, it is interesting that the D19Mit2 marker is closely linked to genes of the two esterase clusters (Fig. 2), some of which may be involved in lipid metabolism. Genome scanning of the RI strains also revealed suggestive but not clearly significant associations between markers on chromosomes 4 and 20 with levels of HDL2 phospholipids and total phospholipids.

To explore for possible genetic relationships between blood pressure and lipid phenotypes in the rat, we have compared the map positions of the QTLs influencing serum lipid phenotypes with those of QTLs we have previously linked to the regulation of blood pressure in the HXB and BXH RI strains. The D19Mit2 marker that is linked to the regulation of basal levels of HDL2 cholesterol maps to a broad region of chromosome 19 previously linked to inherited variation in systolic pressure (12). The region of chromosome 4 that includes the marker for Il6 and that shows suggestive linkage to HDL2 phospholipid levels was previously found to contain a QTL regulating diastolic blood pressure (12). The region of chromosome 20 that includes the D20Utr1 marker and that shows suggestive linkage to HDL2 phospholipid levels after cholesterol feeding has also been reported to contain a QTL regulating blood pressure (11). Finally, comparisons of the SHR.Lx and SHR.1N congenic strains with the SHR progenitor strain, indicate that after cholesterol-rich diet, QTLs modulating cholesterol and phospholipid lipoprotein fractions and total phospholipids are located in segments of chromosomes 8 and 20 (Table III). The results of preliminary blood pressure measurements in these congenic strains have indicated that these chromosome segments also contain genes regulating blood pressure $(11,31)$. Taken together, the current findings suggest that blood pressure and certain serum lipid phenotypes can be influenced either by linked genes or perhaps by common genes with pleiotropic effects. Although it is difficult to distinguish between these two possibilities using RI strains or conventional mapping populations (37), further studies in congenic lines might be useful for separating linked genes that independently regulate blood pressure and lipid metabolism.

Recently, Purcell-Huynh et al. (38) described a QTL on mouse chromosome 17 near the major histocompatibility complex that regulates HDL cholesterol concentration. This region of the mouse genome is homologous to the region of rat chromosome 20 in the SHR.1N congenic strain that we have found to contain a QTL influencing HDL2 phospholipid and LDL/HDL1 cholesterol metabolism (Table III). It is interesting to note that the Clps gene coding for pancreatic colipase is located in this region of mouse chromosome 17 (39). Pancreatic colipase is a protein cofactor for pancreatic lipase and is absolutely necessary for intestinal fat digestion (39, 40). Recent studies in transgenic mice have also indicated that Apoc3 and Apoal on mouse chromosome 9 can be important genetic determinants of lipid metabolism in which Apoal appears to influence especially the HDL cholesterol levels $(41,42)$. The Apoc3 and Apoal genes in the rat map to the differential segment of chromosome 8 where we have mapped QTLs influencing IDL and HDL2 cholesterol levels in the SHR.Lx congenic strain. Finally, studies in gene targeted mice have demonstrated that inactivation of the hepatic lipase (Lipc) gene on mouse chromosome 9 can result in mild dyslipidemia $(43,44)$. Based on somatic cell hybrid analysis (our unpublished observations) and comparative mapping of conserved blocks of homology in this chromosome region, it is possible that the differential segment of chromosome 8 in the SHR.Lx congenic strain also contains the Lipc gene.

In humans with Syndrome $\mathrm{X}$ or with FDH, hypertension is associated with increased levels of LDL cholesterol and decreased levels of HDL cholesterol. This suggests that in certain human populations: $(a)$ some loci with alleles promoting increased blood pressure may be in linkage disequilibrium with those promoting dyslipidemia; and/or $(b)$ blood pressure and lipid levels may be influenced by pleiotropic effects of the same genes. In this study, the SHR progenitor strain exhibited 
lower levels of total cholesterol and phospholipids, lower levels of VLDL cholesterol and phospholipids, and higher levels of HDL2 phospholipids than the normotensive BN.Lx progenitor strain after feeding a high fat diet (Table I). Thus, when compared with the normotensive BN.Lx strain, the SHR does not fit the pattern of disordered lipid metabolism and hypertension typical of Syndrome X or FDH. However, this does not exclude the possibility that in the SHR some loci with alleles promoting increased blood pressure are linked or even identical to some loci with alleles promoting dyslipidemia. Since blood pressure and lipid levels are multifactorial traits, simple comparisons between genetically dissimilar hypertensive and normotensive strains cannot be used to draw conclusions about potential linkage relationships between genetic factors influencing these complex phenotypes. In contrast, the blood pressure and lipid patterns in the congenic strains can be much more informative with respect to such linkage relationships because each congenic strain differs from the SHR progenitor with respect to only a single chromosome segment. Thus, it should be noted that the SHR progenitor strain exhibits higher blood pressure, higher levels of IDL and LDL/HDL1 cholesterol, and lower levels of HDL2 cholesterol than the SHR.Lx and SHR.1N congenic strains. These findings suggest that in at least some chromosome regions, the SHR progenitor has simultaneously fixed alleles promoting both hypertension and dyslipidemia in a pattern similar to that proposed for humans with Syndrome X or FDH.

In summary, we have mapped QTLs regulating selected cholesterol and phospholipid phenotypes in the rat to regions of chromosomes 8, 19, and 20 that are suspected to contain genes regulating blood pressure. These findings are consistent with the suggestion that lipid levels and blood pressure may be modulated by linked genes or perhaps even the same genes. To the extent that homologous regions of the human genome also contain genes regulating lipid metabolism and blood pressure, the current findings would provide support for the idea that in patients with essential hypertension genetic factors contribute to the frequent association of dyslipidemia and increased blood pressure. It remains to be determined whether blood pressure and cholesterol phenotypes cosegregate due to a linkage of different genes or even due to pleiotropic effects of common genes.

\section{Acknowledgments}

This work was supported by grant A7011505 from the Academy of Sciences of the Czech Republic and by a grant from the PECO Program of the European Commission (EURHYPGEN Project). The research of M. Pravenec was supported in part by an International Research Scholar's Award from the Howard Hughes Medical Institute. This work was also supported by a grant from the National Institutes of Health (Hypertension Program Project PO1 HL-35018) and by funds provided by the Cigarette and Tobacco Surtax Fund of the State of California through the Tobacco Related Diseases Research Program of the University of California, Grant 4RT-0362.

\section{References}

1. Reaven, G.M. 1995. Pathophysiology of insulin resistance in human disease. Physiol. Rev. 75:473-486.

2. Zavaroni, I., S. Mazza, E. Dall'aglio, P. Gasparini, M. Passeri, and G.M. Reaven. 1992. Prevalence of hyperinsulinemia in patients with high blood pressure. J. Intern. Med. 231:235-240.

3. Reaven, G.M., and Y.-D.I. Chen. 1988. Role of insulin in regulation of li- poprotein metabolism in diabetes. Diabetes Metab. Rev. 4:639-652.

4. Williams, R.R., S.C. Hunt, P.N. Hopkins, B.M. Stults, L.L. Wu, S.J. Hasstedt, G.K. Barlow, S.H. Stephenson, J.M. Lalouel, and H. Kuida. 1988. Familial dyslipidemic hypertension. Evidence from 58 Utah families for a syndrome present in approximately $12 \%$ of patients with essential hypertension. J. Am. Med. Assoc. 259:3579-3586.

5. Williams, R.R., S.C. Hunt, P.N. Hopkins, L.L. Wu, and J.M. Lalouel. 1994. Evidence for single gene contributions to hypertension and lipid disturbances: definition, genetics, and clinical significance. Clin. Genet. 46:80-87.

6. Reaven, G.M., and H. Chang. 1991. Relationship between blood pressure, plasma insulin and triglyceride concentration, and insulin action in spontaneous hypertensive and Wistar-Kyoto rats. Am. J. Hypertens. 4:34-38.

7. Reaven, G.M., J. Twersky, and H. Chang. 1991. Abnormalities in carbohydrate and lipid metabolism in Dahl rats. Hypertension (Dallas). 18:630-635.

8. Dall'Aglio, E., P. Tosini, P. Ferrari, I. Zavaroni, M. Passeri, and G.M Reaven. 1991. Abnormalities of insulin and lipid metabolism in Milan hypertensive rats. Am. J. Hypertens. 4:773-775.

9. Vincent, M., E.H. Boussairi, R. Cartier, M. Lo, A. Sassolas, C. Cerutti, C. Barres, M.-P. Gustin, G. Cuisinaud, N.J. Samani, et al. 1993. High blood pressure and metabolic disorders are associated in the Lyon hypertensive rat. J. Hypertens. 11:1179-1185.

10. Thorburn, A.W., L.H. Storlein, and A.B. Jenkins. 1989. Fructose-induced in vitro insulin resistance and elevated plasma triglyceride levels in rats. Am. J. Clin. Nutr. 49:1153-1163.

11. Pravenec, M., P. Klír, V. Kren, J. Zicha, and J. Kunes. 1989. An analysis of spontaneous hypertension in spontaneously hypertensive rats by means of new recombinant inbred strains. J. Hypertens. 7:217-222.

12. Pravenec, M., D. Gauguier, J.-J. Schott, J. Buard, V. Kren, V. Bílá, C. Szpirer, J. Szpirer, J.-M. Wang, H. Huang, et al. 1995. Mapping of quantitative trait loci for blood pressure and cardiac mass in the rat by genome scanning of recombinant inbred strains. J. Clin. Invest. 96:1973-1978.

13. Kren, V. 1975. Genetics of the polydactyly-luxate syndrome in the Norway rat, Rattus norvegicus. Acta Univ. Carol. Med. Monogr. 68:1-103.

14. Kren, V., D. Krenová, M. Pravenec, and M. Zdobinská. 1995. Chromosome 8 congenic strains: tools for genetic analysis of limb malformation, plasma triglycerides, and blood pressure in the rat. Folia Biol. (Prague). 41:284-292.

15. Vrána, A., I. Kazdová, Z. Dobesová, J. Kunes, V. Kren, V. Bílá, P. Stolba, and I. Klimes. 1993. Triglyceridemia, glucoregulation, and blood pressure in various rat strains. Ann. NY Acad. Sci. 63:57-68.

16. Serikawa, T., T. Kuramoto, P. Hilbert, M. Mori, J. Yamada, C.J. Dubay, K. Lindpainter, D. Ganten, J.L. Guenet, G.M. Lathrop, and J.S. Beckmann. 1992. Rat gene mapping using PCR-analyzed microsatellites. Genetics. 131:701721

17. Jacob, H., D. Brown, R. Bunker, M. Daly, V. Dzau, A. Goodman, G. Koike, V. Kren, T. Kurtz, A. Lernmark, et al. 1995. A genetic linkage map of the laboratory rat (Rattus norvegicus). Nat. Genet. 9:63-69.

18. Mathern, P., E.A. Goldmuntz, Y. Du, H. Zha, J.M. Cash, L.J. Crofford, R.L. Wilder, and E.F. Remmers. 1994. Nine polymorphic markers characterized by polymerase chain reaction techniques form two linkage groups on rat chromosome 8. Cytogenet. Cell Genet. 66:283-286.

19. Remmers, E.F., Y. Du, H. Zha, E.A. Goldmuntz, and R.L. Wilder. 1995. Ten polymorphic DNA loci, including five in the rat MHC (RT1) region, form a single linkage group on rat chromosome 20. Immunogenetics. 41:316-319.

20. Bottger, A., M. den Bieman, A. Lankhorst, H.A. van Lith, and L.F.M. van Zutphen. 1996. Strain-specific response to hypercholesterolaemic diets in the rat. Lab. Anim. 30:149-157.

21. Terpstra, A.H.M., C.J.H. Woodward, and F.J. Sanchez-Muniz. 1981. Improved techniques for the separation of serum lipoproteins by density gradient ultracentrifugation: visualization by prestaining and rapid isolation of serum lipoproteins from small volumes of serum. Anal. Biochem. 111:149-157.

22. Pravenec, M., D. Gauguier, J.-J. Schott, J. Buard, V. Kren, V. Bílá, C. Szpirer, J. Szpirer, J.-M. Wang, H. Huang, et al. 1995. A genetic linkage map of the rat derived from recombinant inbred strains. Mamm. Genome. 7:117-127.

23. Otsen, M., M. den Bieman, M.T.R. Kuiper, M. Pravenec, V. Kren, T.W. Kurtz, H.J. Jacob, A.E. Lankhorst, and L.F.M. van Zutphen. 1995. Construction of a genetic linkage map in the rat using AFLP ${ }^{\mathrm{TM}}$ markers. In Use of DNA Markers for Genetic Characterization and Linkage Studies in the Rat (Rattus norvegicus). M. Otsen thesis, Utrecht University. 79-98.

24. Brault, D., L. Noe, J. Etienne, J. Hamelin, A. Raisonnier, A. Souli, J. Chuat, J. Claude, I. Dugail, A. Quignard-Boulange, et al. 1992. Sequence of rat lipoprotein lipase-encoding cDNA. Gene (Amst.). 121:237-246.

25. Meroni, G., N. Malgaretti, P. Magnaghi, and R. Taramelli. 1990. Nucleotide sequence of the cDNA for lecithin-cholesterol acyl transferase (LCAT) from the rat. Nucleic Acids Res. 18:5308.

26. McLean, J., K. Wion, D. Drayna, C. Fielding, and R. Lawn. 1986. Human lecithin-cholesterol acyltransferase gene: complete gene sequence and sites of expression. Nucleic Acids Res. 14:9397-9406.

27. Manly, K.F. 1993. A Macintosh program for storage and analysis of experimental mapping data. Mamm. Genome. 4:303-313.

28. Belknap, J.K. 1992. Empirical estimates of Bonferroni corrections for use in chromosome mapping studies with the BXD recombinant inbred strains. Behav. Genet. 22:677-684. 
29. Neumann, P.E. 1992. Inference in linkage analysis of multifactorial traits using recombinant inbred strains of mice. Behav. Genet. 22:665-675.

30. Warden, C.H., C.A. Langner, J.I. Gordon, B.A. Taylor, J.W. McLean, and A.J. Lusis. 1989. Tissue-specific expression, developmental regulation, and chromosomal mapping of the lecithin:cholesterol acyltransferase gene. J. Biol. Chem. 264:21573-21581.

31. Lu, S., M. Pravenec, V. Kren, J.-M. Wang, and T.W. Kurtz. 1995. The SHR.Lx congenic strain delineates a contribution of chromosome 8 gene(s) to hypertension in the spontaneously hypertensive rat. Hypertension (Dallas). 26: 538a. (Abstr.)

32. Scherer, G., E. Bausch, A. Gaa, and O. von Deimling. 1989. Gene mapping on chromosome 8 by interspecific crosses: new data on a linkage group conserved on human chromosome 16q. Genomics. 5:275-282.

33. Azoulay, M., I. Henry, F. Tata, D. Weil, K.H. Grzeschik, M.E. Chaves, N. McIntyre, R. Williamson, S.E. Humphries, and C. Junien. 1987. The structural gene for lecithin:cholesterol acyl transferase (LCAT) maps to 16q22. Ann. Hum. Genet. 51:129-136.

34. Kirchgessner, T.G., R.C. LeBoeuf, C.A. Lanoner, S. Zollman, C.H Chang, B.A. Taylor, M.C. Schotz, J.I. Gordon, and A.J. Lusis. 1989. Genetic and developmental regulation of the lipoprotein lipase gene: loci both distal and proximal to the lipoprotein lipase structural gene control enzyme expression. J. Biol. Chem. 264:1473-1482.

35. Pulcini, T., P. Terru, J.T. Sparrow, H.J. Pownall, and G. Ponsin. 1995. Plasma factors affecting the in vitro conversion of high-density lipoproteins labeled with a non-transferable marker. Biochim. Biophys. Acta. 1254:13-21.

36. Mehlum, A., B. Staels, N. Duverger, A. Tailleux, G. Castro, C. Fievet, G. Luc, J. C. Fruchart, G. Olivecrona, G. Skretting, et al. 1995. Tissue-specific expression of the human gene for lecithin:cholesterol acyltransferase in trans- genic mice alters blood lipids, lipoproteins and lipases towards a less atherogenic profile. Eur. J. Biochem. 230:567-575.

37. Darvasi, A., and M. Soller. 1995. Advanced intercross lines, an experimental population for fine genetic mapping. Genetics. 141:1199-1207.

38. Purcell-Huynh, D.A., A. Weinreb, L.W. Castellani, M. Mehrabian, M.H. Doolittle, and A.J. Lusis. 1995. Genetic factors in lipoprotein metabolism. Analysis of a genetic cross between inbred mouse strains NZB/BINL and SM/J using a complete linkage map approach. J. Clin. Invest. 96:1845-1858.

39. Warden, C.H., R.C. Davis, M.Y. Yoon, D.Y. Hui, K. Svenson, Y.R. Xia A. Diep, K.Y. He, and A.J. Lusis. 1993. Chromosomal localization of lipolytic enzymes in the mouse: pancreatic lipase, colipase, hormone-sensitive lipase, hepatic lipase, and carboxyl ester lipase. J. Lipid Res. 34:1451-1455.

40. Erlanson-Albertsson, C. 1992. Pancreatic colipase. Structural and physiological aspects. Biochim. Biophys. Acta. 1125:1-7.

41. Reaven, G.M., C.E. Mondon, Y.D. Chen, and J.L. Breslow. 1994. Hypertriglyceridemic mice transgenic for the human apolipoprotein C-III gene are neither insulin-resistant nor hyperinsulinemic. J. Lipid Res. 35:820-824.

42. Chajek-Shaul, T., T. Hayek, A. Walsh, and J.L. Breslow. 1991. Expression of the human apolipoprotein A-1 gene in transgenic mice alters high den sity lipoprotein (HDL) particle size distribution and diminishes selective uptake of HDL cholesteryl esters. Proc. Natl. Acad. Sci. USA. 88:6731-6735.

43. Homanics, G.E., H.V. de Silva, J. Osada, S.H. Zhang, H. Wong, J. Borensztajn, and N. Maeda. 1995. Mild dyslipidemia in mice following targeted inactivation of the hepatic lipase gene. J. Biol. Chem. 270:2974-2980.

44. Cohen, J.C., Z. Wang, S.M. Grundy, M.R. Stosz, and R. Guerra. 1994. Variation at the hepatic lipase and apolipoprotein AI/CIII/AIV loci is a major cause of genetically determined variation in plasma HDL cholesterol levels. $J$. Clin. Invest. 94:2377-2384. 